\title{
Uniqueness of L-Functions Concerning Certain Differential Polynomials
}

\author{
Wen-Jie Hao $\mathbb{D}^{1}$ and Jun-Fan Chen $\mathbb{D}^{1,2}$ \\ ${ }^{1}$ Department of Mathematics, Fujian Normal University, Fuzhou 350117, China \\ ${ }^{2}$ Key Laboratory of Applied Mathematics (Putian University), Fujian Province University, Fujian Putian 351100, China
}

Correspondence should be addressed to Jun-Fan Chen; junfanchen@163.com

Received 21 June 2018; Accepted 18 September 2018; Published 18 October 2018

Academic Editor: Cengiz Çinar

Copyright ( $) 2018$ Wen-Jie Hao and Jun-Fan Chen. This is an open access article distributed under the Creative Commons Attribution License, which permits unrestricted use, distribution, and reproduction in any medium, provided the original work is properly cited.

\begin{abstract}
Relying on Nevanlinna theory and the properties of L-functions in the extended Selberg class, we mainly study the uniqueness problems on L-functions concerning certain differential polynomials. This generalizes some results of Steuding, Li, Fang, and LiuLi-Yi.
\end{abstract}

\section{Introduction}

The Riemann hypothesis as one of the millennium problems has been given a lot of attention by mathematical workers for a long time. Selberg guessed that the Riemann hypothesis is also true for L-functions in the Selberg class. Such an Lfunction based on Riemann zeta function as the prototype is defined to be a Dirichlet series

$$
L(s)=\sum_{n=1}^{\infty} \frac{a(n)}{n^{s}}
$$

of a complex variable $s=\sigma+i t$ satisfying the following axioms:

(i) Ramanujan hypothesis: $a(n) \ll n^{\varepsilon}$ for every $\varepsilon>0$

(ii) Analytic continuation: there is a nonnegative integer $m$ such that $(s-1)^{m} L(s)$ is an entire function of finite order of type

(iii) Functional equation: $L$ satisfies a functional equation

$$
\Lambda_{L}(s)=\omega \overline{\Lambda_{L}(1-\bar{s})}
$$

where

$$
\Lambda_{L}(s)=L(s) Q^{s} \prod_{j=1}^{K} \Gamma\left(\lambda_{j} s+v_{j}\right)
$$

with positive real numbers $\mathrm{Q}, \lambda_{j}$, and complex numbers $\nu_{j}, \omega$ with $\operatorname{Re} v_{j} \geq 0$ and $|\omega|=1$

(iv) Euler product: $\log L(s)=\sum_{n=1}^{\infty}\left(b(n) / n^{s}\right)$, where $b(n)=$ 0 unless $n$ is a positive power of a prime and $b(n) \ll n^{\theta}$ for some $\theta<1 / 2$

It is mentioned that there are many Dirichlet series only satisfying axioms (i)-(iii) [1] and are regarded as the extended Selberg class. All the L-functions are studied in this article from the extended Selberg class. Therefore, the conclusions obtained in this article are also true for L-functions in the Selberg class. The uniqueness of two L-functions was firstly studied by Steuding [2], as seen from Theorem 1 .

Theorem 1 (see [2]). Suppose thatc is a finite complex number. If two $L$-functions $L_{1}$ and $L_{2}$ with $a(1)=1$ share $c C M$, then $L_{1} \equiv L_{2}$.

In 2016, $\mathrm{Hu}$ and $\mathrm{Li}$ [3] gave an example $L_{1}=1+2 / 4^{s}$ and $L_{2}=1+3 / 9^{s}$. From this we can know the above theorem is false when $c=1$.

Due to the complication to study the distribution of public zero of two L-functions, researchers take up study of the relationship of an L-function and a meromorphic function. Since L-function itself can be analytically continued as a meromorphic function in the whole complex plane, therefore, L-functions will be taken as special meromorphic 
functions, with the help of Nevanlinna's value distribution theory, in order to study the uniqueness of L-functions. Suppose that $F$ and $G$ are two nonconstant meromorphics in the whole complex plane; $c$ denotes a values in the extended complex plane. If $F-c$ and $G-c$ have the same zeros counting multiplicities, we say that $F$ and $G$ share $c$ CM. If $F-c$ and $G-c$ have the same zeros ignoring multiplicities, then we say that $F$ and $G$ share $c$ IM. One nonconstant meromorphic function in the whole complex plane can be determined by five such preimages or four such preimages $[4,5]$. In 2010, Li [6] considered a meromorphic function and a nonconstant L-function and he obtained the following.

Theorem 2 (see [6]). Let $a$ and $b$ be two distinct finite values and $f$ be a meromorphic function in the complex plane with finitely many poles. If $f$ and a nonconstant $L$-function $L$ share a CM and $b$ IM, then $L \equiv f$.

In 1997, the following question was raised by Lahiri [7]: what is the relationship between function $f$ and function $g$, when two differential polynomials have the same nonzero finite value? The two differential polynomials are generated by $f$ and $g$, respectively. In this direction, Fang [8] proved the following theorem.

Theorem 3 (see [8]). Let $f$ and $g$ be two nonconstant entire functions, and let $n, k$ be two positive integers. Suppose that $\left[f^{n}(f-1)\right]^{(k)}$ and $\left[g^{n}(g-1)\right]^{(k)}$ share 1 CM. If $n \geq 2 k+8$, then $f \equiv g$.

Recently, Liu-Li-Yi $[9,10]$ considered an L-function and a meromorphic function whose certain differential polynomials share one finite nonzero value. The following conclusions were obtained.

Theorem 4 (see [9]). Let $f$ be a nonconstant meromorphic function, let $L$ be an $L$-function, and let $n$ and $k$ be two positive integers. Suppose that $\left(f^{n}\right)^{(k)}$ and $\left(L^{n}\right)^{(k)}$ share 1 CM. If $n>$ $3 k+6$, then $f \equiv t L$ for a constant $t$ satisfying $t^{n}=1$.

Theorem 5 (see [10]). Let $f$ be a nonconstant meromorphic function, let $L$ be an L-function, and let $n$ and $k$ be two positive integers. Suppose that $\left[f^{n}(f-1)\right]^{(k)}$ and $\left[L^{n}(L-1)\right]^{(k)}$ share 1 $C M$. If $n>3 k+9$ and $k \geq 2$, then $f \equiv L$.

Naturally, is it still set up if it can be generalized to the general differential polynomials, for instance, $\left[f^{n}\left(f^{m}-1\right)\right]^{(k)}$ or $\left[f^{n}(f-1)^{m}\right]^{(k)}$ ? For simplicity, we use the notations $d=$ $\operatorname{GCD}(n, m)$ and $\widetilde{m}:=\widetilde{m}(\alpha)$, where

$$
\widetilde{m}(\alpha)= \begin{cases}0, & \alpha=0 \\ m, & \alpha \neq 0\end{cases}
$$

In this paper, we have the results as follows.

Theorem 6. Let $f$ be a nonconstant meromorphic function, let $L$ be an $L$-function, and let $n, m$, and $k$ be three positive integers and $\alpha, \beta$ be two constants satisfying $|\alpha|+|\beta| \neq 0$. Suppose that $\left[f^{n}\left(\alpha f^{m}+\beta\right)\right]^{(k)}$ and $\left[L^{n}\left(\alpha L^{m}+\beta\right)\right]^{(k)}$ share 1 CM. If $n>$ $3 k+\widetilde{m}+6$, then $f \equiv t L$, where

(i) when $\alpha \beta=0$, $t$ is a constant such that $t^{n+\widetilde{m}}=1$

(ii) when $\alpha \beta \neq 0, k \geq 2, t$ is a constant such that $t^{d}=1$

Remark 7. In Theorem 6, if $\alpha=0, \beta=1$, we can get Theorem 4. If $\alpha=m=1, \beta=-1$, we can get Theorem 5 . Moreover, if the condition $n>3 k+7$ substitutes $n>$ $3 k+9$ in Theorem 5 , then we can obtain $f \equiv L$, which implies the conclusion remains valid in Theorem 5 . Therefore, Theorem 6 is the generalization of Theorems 4 and 5 .

Remark 8. In Theorem 6 , the condition $n>3 k+\widetilde{m}+6$ cannot be dropped. Let $\alpha=0, \beta=1, f=2 \zeta-z, L=\zeta, n=1, k=1$. Then $f^{\prime}-1=2 \zeta^{\prime}-2, L^{\prime}-1=\zeta^{\prime}-1$. This shows that $f^{\prime}$ and $L^{\prime}$ share $1 \mathrm{CM}$. But $f \not \equiv L$.

Theorem 9. Let $f$ be a nonconstant meromorphic function, let $L$ be an $L$-function, and let $n, m$, and $k$ be three positive integers. Suppose that $\left[f^{n}(f-1)^{m}\right]^{(k)}$ and $\left[L^{n}(L-1)^{m}\right]^{(k)}$ share 1 CM. If $n>3 k+m+6$ and $k \geq 2$, then $f \equiv L$ or $f^{n}(f-1)^{m} \equiv L^{n}(L-1)^{m}$.

Remark 10. In Theorem 9, if $m=1$, we can get Theorem 5 . Actually, if $f \not \equiv L$ and $f^{n}(f-1) \equiv L^{n}(L-1)$, we can get a contradiction by subcase 3.2 in the proof of Theorem 6 . We can also see that Theorem 5 is included in Theorem 9.

Moreover, we consider what will happen if the CM becomes the IM in Theorems 6 and 9. The theorems are then established.

Theorem 11. Let $f$ be a nonconstant meromorphic function, let $L$ be an L-function, and let $n, m$, and $k$ be three positive integers and $\alpha, \beta$ be two constants satisfying $|\alpha|+|\beta| \neq 0$. Suppose that $\left[f^{n}\left(\alpha f^{m}+\beta\right)\right]^{(k)}$ and $\left[L^{n}\left(\alpha L^{m}+\beta\right)\right]^{(k)}$ share 1 IM. If $n>7 k+4 \widetilde{m}+11$, then $f \equiv t L$, where

(i) when $\alpha \beta=0$, $t$ is a constant such that $t^{n+\widetilde{m}}=1$

(ii) when $\alpha \beta \neq 0, k \geq 2, t$ is a constant such that $t^{d}=1$

Theorem 12. Let $f$ be a nonconstant meromorphic function, let $L$ be an L-function, and let $n, m$, and $k$ be three positive integers. Suppose that $\left[f^{n}(f-1)^{m}\right]^{(k)}$ and $\left[L^{n}(L-1)^{m}\right]^{(k)}$ share 1 IM. If $n>7 k+4 m+11, k \geq 2$, then $f \equiv \operatorname{Lor} f^{n}(f-1)^{m} \equiv$ $L^{n}(L-1)^{m}$.

Corollary 13. Let $f$ be a nonconstant meromorphic function, let $L$ be an L-function, and let $n, k$ be two positive integers. Suppose that $\left(f^{n}\right)^{(k)}$ and $\left(L^{n}\right)^{(k)}$ share 1 IM. If $n>7 k+11$, then $f \equiv t L$ for a constant $t$ satisfying $t^{n}=1$.

Corollary 14. Let $f$ be a nonconstant meromorphic function, let $L$ be an L-function, and let $n, k$ be two positive integers. Suppose that $\left[f^{n}(f-1)\right]^{(k)}$ and $\left[L^{n}(L-1)\right]^{(k)}$ share 1 IM. If $n>7 k+15$ and $k \geq 2$, then $f \equiv L$. 
To prove the main theorems, the order $\rho(f)$ of a meromorphic function $f$ will be needed. It is defined to be a superior limit

$$
\rho(f)=\limsup _{r \rightarrow \infty} \frac{\log T(r, f)}{\log r} .
$$

Next, we introduce some definitions.

Definition 15. Suppose that $p$ is a positive integer and $c$ is a value in the extended complex plane. Then $N_{p)}(r, 1 /(F-c))$ is defined as the counting function of those zeros of $F-c$ of order $\leq p \cdot N_{(p}(r, 1 /(F-c))$ is defined as the counting function of those zeros of $F-c$ of order $\geq p \cdot \bar{N}_{p)}(r, 1 /(F-c))$, $\bar{N}_{(p}(r, 1 /(F-c))$ are defined as the corresponding reduced counting functions.

Definition 16. Suppose that $z_{0}$ is a common $c$-point of $F$ and $G$ with multiplicity $k$ and $l$, respectively. We denote by $\bar{N}_{L}(r, 1 /(F-c))$ the reduced counting function of those $c$ points of $F$ and $G$ where $k>l$ and denote by $\bar{N}_{E}^{(2}(r, 1 /(F-c))$ the reduced counting function of those $c$-points of $F$ and $G$ where $k=l \geq 2$. Similarly, we can define $\bar{N}_{L}(r, 1 /(G-c))$ and $\bar{N}_{E}^{(2}(r, 1 /(G-c))$.

Definition 17. Suppose that $c$ is a value in the extended complex plane and $k$ is a positive integer. We define

$$
\begin{aligned}
N_{k}\left(r, \frac{1}{F-c}\right)= & \bar{N}\left(r, \frac{1}{F-c}\right)+\bar{N}_{(2}\left(r, \frac{1}{F-c}\right)+\cdots \\
& +\bar{N}_{(k}\left(r, \frac{1}{F-c}\right) .
\end{aligned}
$$

\section{Some Lemmas}

In order to facilitate the proofs of the theorems, we list some important lemmas which will be employed in this paper.

Lemma 18 (see [11]). Let $f$ be a nonconstant meromorphic function and let

$$
F=\frac{\sum_{k=0}^{p} a_{k} f^{k}}{\sum_{j=0}^{q} b_{j} f^{j}}
$$

be an irreducible rational function in $f$ with constant coefficients $\left\{a_{k}\right\}$ and $\left\{b_{j}\right\}$, where $a_{p} \neq 0$ and $b_{q} \neq 0$. Then $T(r, F)=$ $d_{0} T(r, f)+O(1)$, where $d_{0}=\max \{p, q\}$.

Lemma 19 (see [4], Theorem 3.2). Let $f$ be a nonconstant meromorphic function, let $k$ be a positive integer, and let $c$ be a nonzero finite complex number. Then

$$
\begin{aligned}
T(r, f) \leq & \bar{N}(r, f)+N\left(r, \frac{1}{f}\right)+N\left(r, \frac{1}{f^{(k)}-c}\right) \\
& -N\left(r, \frac{1}{f^{(k+1)}}\right)+S(r, f)
\end{aligned}
$$

$$
\begin{aligned}
\leq & \bar{N}(r, f)+N_{k+1}\left(r, \frac{1}{f}\right)+\bar{N}\left(r, \frac{1}{f^{(k)}-c}\right) \\
& -N_{0}\left(r, \frac{1}{f^{(k+1)}}\right)+S(r, f),
\end{aligned}
$$

where $N_{0}\left(r, 1 / f^{(k+1)}\right)$ is the counting function which only counts those points such that $f^{(k+1)}=0$ but $f\left(f^{(k)}-c\right) \neq 0$.

Lemma 20 (see [5]). Let $f$ be a nonconstant meromorphic function and $k$ be a positive integer. Then

$$
N\left(r, \frac{1}{f^{(k)}}\right) \leq N\left(r, \frac{1}{f}\right)+k \bar{N}(r, f)+S(r, f) .
$$

Lemma 21. Let $f$ be a nonconstant meromorphic function, let $L$ be an $L$-function, and let $n, m$, and $k$ be three positive integers and $\alpha, \beta$ be two constants satisfying $|\alpha|+|\beta| \neq 0$. Set

$$
\begin{aligned}
& F=\left[f^{n}\left(\alpha f^{m}+\beta\right)\right]^{(k)}, \\
& G=\left[L^{n}\left(\alpha L^{m}+\beta\right)\right]^{(k)} .
\end{aligned}
$$

If $F$ and $G$ share $1 C M$ and $n>3 k+\widetilde{m}+6$, then $1 /(F-1)=$ $(V G+U-V) /(G-1)$, where $U \neq 0, V$ are two constants.

Proof. Let

$$
\begin{aligned}
& W=\frac{F^{\prime \prime}}{F^{\prime}}-2 \frac{F^{\prime}}{F-1}-\left(\frac{G^{\prime \prime}}{G^{\prime}}-2 \frac{G^{\prime}}{G-1}\right), \\
& F_{1}=f^{n}\left(\alpha f^{m}+\beta\right), \\
& G_{1}=L^{n}\left(\alpha L^{m}+\beta\right) .
\end{aligned}
$$

If $L$ is a constant, we have from (12) that $G_{1}$ is a constant, $G \equiv 0$. Since $F$ and $G$ share $1 \mathrm{CM}$, we obtain $F \neq 1$. Using Lemma 18, we deduce

$$
T\left(r, F_{1}\right)=(n+\widetilde{m}) T(r, f)+S(r, f) .
$$

Moreover, from Lemma 19, we know

$$
\begin{aligned}
T\left(r, F_{1}\right) \leq & \bar{N}\left(r, F_{1}\right)+N_{k+1}\left(r, \frac{1}{F_{1}}\right)+\bar{N}\left(r, \frac{1}{F-1}\right) \\
& +S(r, f) \\
\leq & \bar{N}(r, f)+N_{k+1}\left(r, \frac{1}{f^{n}}\right) \\
& +N_{k+1}\left(r, \frac{1}{\alpha f^{m}+\beta}\right)+S(r, f) \\
\leq & (k+\widetilde{m}+2) T(r, f)+S(r, f) .
\end{aligned}
$$

Combining (13) with $n>3 k+\widetilde{m}+6$, we get that $L$ is a nonconstant L-function. Similarly, using Lemma 18, we deduce

$$
T\left(r, G_{1}\right)=(n+\widetilde{m}) T(r, L)+S(r, L)
$$


Since $\left(F_{1}\right)^{(k)}=F$, we get

$$
m\left(r, \frac{1}{F_{1}}\right) \leq m\left(r, \frac{1}{F}\right)+S(r, f) .
$$

Suppose $W \not \equiv 0, z_{0}$ is a zero of $F-1$ of order $j$, and $z_{0}$ is a zero of $G-1$ of order $j$, in view of $F$ and $G$ sharing 1 CM. By checking the Laurent expansion of $W$, we have $W\left(z_{0}\right)=0$ when $j=1$ and $W\left(z_{0}\right) \neq \infty$ when $j \geq 2$. Thus

$$
\begin{aligned}
N_{1)}\left(r, \frac{1}{F-1}\right) & \left.=N_{1}\right)\left(r, \frac{1}{G-1}\right) \leq N\left(r, \frac{1}{W}\right) \\
& \leq T(r, W)+O(1) \\
& \leq N(r, W)+S(r, f)+S(r, L) .
\end{aligned}
$$

Similarly, assume that $z_{1}$ is a pole of $F$ of order 1 ; we get $W\left(z_{1}\right) \neq \infty$. If $z_{2}$ is a pole of $G$ of order 1 , we get $W\left(z_{2}\right) \neq \infty$. Therefore, we get, by a calculation and (11), that

$$
\begin{aligned}
N(r, W) \leq & \bar{N}_{(2}\left(r, \frac{1}{F}\right)+\bar{N}_{(2}\left(r, \frac{1}{G}\right)+\bar{N}_{(2}(r, F) \\
& +\bar{N}_{(2}(r, G)+\bar{N}_{0}\left(r, \frac{1}{F^{\prime}}\right)+\bar{N}_{0}\left(r, \frac{1}{G^{\prime}}\right) .
\end{aligned}
$$

where $\bar{N}_{0}\left(r, 1 / F^{\prime}\right)$ is the reduced counting function of those zeros of $F^{\prime}$ not that of $F(F-1)$. From the definition, we obtain

$$
\begin{gathered}
\bar{N}_{0}\left(r, \frac{1}{G^{\prime}}\right)+\bar{N}_{(2}\left(r, \frac{1}{G-1}\right)+N_{(2}\left(r, \frac{1}{G}\right) \\
-\bar{N}_{(2}\left(r, \frac{1}{G}\right) \leq N\left(r, \frac{1}{G^{\prime}}\right) .
\end{gathered}
$$

By Lemma 20, we obtain

$$
N\left(r, \frac{1}{G^{\prime}}\right) \leq N\left(r, \frac{1}{G}\right)+\bar{N}(r, G)+S(r, L) .
$$

Combining (19) with (20), we have

$$
\begin{aligned}
& \bar{N}_{0}\left(r, \frac{1}{G^{\prime}}\right)+\bar{N}_{(2}\left(r, \frac{1}{G-1}\right) \\
& \quad \leq \bar{N}\left(r, \frac{1}{G}\right)+\bar{N}(r, G)+S(r, L) .
\end{aligned}
$$

We can get

$$
\bar{N}\left(r, \frac{1}{F-1}\right)=N_{1)}\left(r, \frac{1}{F-1}\right)+\bar{N}_{(2}\left(r, \frac{1}{G-1}\right),
$$

in view of the assumption that $F$ and $G$ share $1 \mathrm{CM}$. Combining (17)-(22), the second fundamental theorem yields

$$
\begin{aligned}
T(r, F) \leq & \bar{N}(r, F)+\bar{N}\left(r, \frac{1}{F}\right)+\bar{N}\left(r, \frac{1}{F-1}\right) \\
& -N_{0}\left(r, \frac{1}{F^{\prime}}\right)+S(r, f) \\
\leq & \bar{N}(r, F)+\bar{N}\left(r, \frac{1}{F}\right)+\bar{N}_{(2}\left(r, \frac{1}{F}\right)
\end{aligned}
$$

$$
\begin{aligned}
& +\bar{N}_{(2}\left(r, \frac{1}{G}\right)+\bar{N}_{(2}(r, F)+\bar{N}_{(2}(r, G) \\
& +\bar{N}\left(r, \frac{1}{G}\right)+\bar{N}(r, G)+S(r, f) \\
& +S(r, L) .
\end{aligned}
$$

From the first fundamental theorem, we know

$$
\begin{aligned}
T\left(r, \frac{1}{F}\right) \leq & N_{2}(r, F)+N_{2}(r, G)+N_{2}\left(r, \frac{1}{F}\right) \\
& +N_{2}\left(r, \frac{1}{G}\right)+S(r, f)+S(r, L),
\end{aligned}
$$

where

$$
\begin{aligned}
& N_{2}\left(r, \frac{1}{F}\right)=N\left(r, \frac{1}{F}\right) \\
&- {\left[N_{(2}\left(r, \frac{1}{F}\right)-2 \bar{N}_{(2}\left(r, \frac{1}{F}\right)\right], } \\
& N_{2}\left(r, \frac{1}{G}\right)=N\left(r, \frac{1}{G}\right) \\
&- {\left[N_{(2}\left(r, \frac{1}{G}\right)-2 \bar{N}_{(2}\left(r, \frac{1}{G}\right)\right] . }
\end{aligned}
$$

Let $z_{3}$ be a zero of $f$ of order $i, i \geq 1$. Since $n>3 k+\widetilde{m}+6>$ $k+2, n i-k>(k+2) i-k=(i-1) k+2 i \geq 2$, we can deduce the zeros of $F$ of order $\geq 3$. Thus

$$
N_{(2}\left(r, \frac{1}{F}\right)-2 \bar{N}_{(2}\left(r, \frac{1}{F}\right) \geq(n-k-2) N\left(r, \frac{1}{f}\right) .
$$

Also, for $G$, we can deduce that

$$
N_{(2}\left(r, \frac{1}{G}\right)-2 \bar{N}_{(2}\left(r, \frac{1}{G}\right) \geq(n-k-2) N\left(r, \frac{1}{L}\right) .
$$

Combining (24)-(27), we get

$$
\begin{aligned}
T\left(r, \frac{1}{F}\right)-N\left(r, \frac{1}{F}\right) \\
\leq N\left(r, \frac{1}{G}\right)+N_{2}(r, F)+N_{2}(r, G) \\
\quad-(n-k-2) N\left(r, \frac{1}{f}\right)-(n-k-2) N\left(r, \frac{1}{L}\right) \\
\quad+S(r, f)+S(r, L) .
\end{aligned}
$$

By Lemma 20, we obtain

$$
N\left(r, \frac{1}{G}\right) \leq N\left(r, \frac{1}{G_{1}}\right)+k \bar{N}\left(r, G_{1}\right)+S(r, L) .
$$

Using the first fundamental theorem, we have

$$
\begin{aligned}
T\left(r, F_{1}\right) & =T\left(r, \frac{1}{F_{1}}\right)+O(1) \\
& =m\left(r, \frac{1}{F_{1}}\right)+N\left(r, \frac{1}{F_{1}}\right)+O(1) .
\end{aligned}
$$


From(13), (16), (28)-(30), we get

$$
\begin{aligned}
& (n+\widetilde{m}) T(r, f) \\
& \leq N\left(r, \frac{1}{F_{1}}\right)+N\left(r, \frac{1}{G_{1}}\right)+k \bar{N}\left(r, G_{1}\right) \\
& +N_{2}(r, F)+N_{2}(r, G)-(n-k-2) N\left(r, \frac{1}{f}\right) \\
& -(n-k-2) N\left(r, \frac{1}{L}\right)+S(r, f)+S(r, L) \\
& \leq n N\left(r, \frac{1}{f}\right)+N\left(r, \frac{1}{\alpha f^{m}+\beta}\right)+n N\left(r, \frac{1}{L}\right) \\
& +N\left(r, \frac{1}{\alpha L^{m}+\beta}\right)+k \bar{N}\left(r, G_{1}\right)+N_{2}(r, F) \\
& +N_{2}(r, G) \\
& -(n-k-2)\left[N\left(r, \frac{1}{f}\right)+N\left(r, \frac{1}{L}\right)\right] \\
& +S(r, f)+S(r, L) \\
& \leq N\left(r, \frac{1}{\alpha f^{m}+\beta}\right)+N\left(r, \frac{1}{\alpha L^{m}+\beta}\right) \\
& +k \bar{N}\left(r, G_{1}\right)+N_{2}(r, F)+N_{2}(r, G) \\
& +(k+2) N\left(r, \frac{1}{f}\right)+(k+2) N\left(r, \frac{1}{L}\right) \\
& +S(r, f)+S(r, L) \text {. }
\end{aligned}
$$

Similarly, we have

$$
\begin{aligned}
(n+\widetilde{m}) T(r, L) \leq & N\left(r, \frac{1}{\alpha f^{m}+\beta}\right) \\
& +N\left(r, \frac{1}{\alpha L^{m}+\beta}\right)+k \bar{N}\left(r, F_{1}\right) \\
& +N_{2}(r, F)+N_{2}(r, G) \\
& +(k+2) N\left(r, \frac{1}{f}\right) \\
& +(k+2) N\left(r, \frac{1}{L}\right)+S(r, f) \\
& +S(r, L) .
\end{aligned}
$$

Since $L$ at most has one pole, we know $N(r, L)=O(\log r)$. At the same time, we know $\bar{N}(r, F)=\bar{N}\left(r, F_{1}\right)=\bar{N}(r, f)$, $\bar{N}(r, G)=\bar{N}\left(r, G_{1}\right)=\bar{N}(r, L), N_{2}(r, F) \leq 2 \bar{N}(r, F)$. Вy $(31)$ and (32), we can obtain the following results, respectively:

$$
\begin{aligned}
(n+\widetilde{m}) T(r, f) \leq & (k+\widetilde{m}+4) T(r, f) \\
& +(k+\widetilde{m}+2) T(r, L) \\
& +S(r, f)+S(r, L),
\end{aligned}
$$

$$
\begin{aligned}
(n+\widetilde{m}) T(r, L) \leq & (2 k+\widetilde{m}+4) T(r, f) \\
& +(k+\widetilde{m}+2) T(r, L) \\
& +S(r, f)+S(r, L) .
\end{aligned}
$$

Assume that there exists some subset $E \subseteq R^{+}$with its linear measure mes $E=\infty$ satisfying $T(r, L) \leq T(r, f)$, as $r \in E$ and $r \longrightarrow \infty$. Then it follows from (33) that $n \leq 2 k+\widetilde{m}+6$, which contradicts $n>3 k+\widetilde{m}+6$. Assume that there exists some subset $E \subseteq R^{+}$with its linear measure mesE $=\infty$ such that $T(r, f) \leq T(r, L)$, as $r \in E$ and $r \longrightarrow \infty$. Then it follows from (34) that $n \leq 3 k+\widetilde{m}+6$, which contradicts $n>3 k+\widetilde{m}+6$. Therefore, $W \equiv 0$. That is,

$$
\frac{F^{\prime \prime}}{F^{\prime}}-2 \frac{F^{\prime}}{F-1}=\frac{G^{\prime \prime}}{G^{\prime}}-2 \frac{G^{\prime}}{G-1} .
$$

Integrating this gives $1 /(F-1)=(V G+U-V) /(G-1)$, where $U \neq 0, V$ are two constants.

This completes the proof of Lemma 21.

Remark 22. Suppose that $F=\left[f^{n}\left(\alpha f^{m}+\beta\right)\right]^{(k)}$ and $G=$ $\left[L^{n}\left(\alpha L^{m}+\beta\right)\right]^{(k)}$ are replaced by $F=\left[f^{n}(f-1)^{m}\right]^{(k)}$ and $G=\left[L^{n}(L-1)^{m}\right]^{(k)}$, respectively, in Lemma 21. That is still true. In fact, $N\left(r, 1 /\left(\alpha f^{m}+\beta\right)\right)$ is replaced by $N\left(r, 1 /(f-1)^{m}\right)$ in (31) and (32); we still get $m T(r, f)$ in (33) and (34).

Lemma 23 (see [12], Theorem 1.2). Suppose that $f$ is a meromorphic of finite order in the complex plane and that $f^{(k)}$ has finitely many zeros for some $k \geq 2$. Then $f$ has finitely many poles in the complex plane.

Lemma 24 (see [4]). Let $f$ be a transcendental meromorphic function, and let $a_{1}, a_{2}$ be two distinct meromorphic functions such that $T\left(r, a_{i}\right)=S(r, f), i=1,2$. Then

$$
\begin{aligned}
T(r, f) \leq & \bar{N}(r, f)+\bar{N}\left(r, \frac{1}{f-a_{1}}\right) \\
& +\bar{N}\left(r, \frac{1}{f-a_{2}}\right)+S(r, f) .
\end{aligned}
$$

Lemma 25 (see [13]). Let $s>0$ and $t$ be relatively prime integers, and let $c$ be a finite complex number such that $c^{s}=1$. Then there exists one and only one common zero of $\omega^{s}-1$ and $\omega^{t}-c$.

Lemma 26 (see $[14,15]$ ). Let $f$ be a nonconstant meromorphic function, and let $p, k$ be two positive integers. Then

$$
N_{p}\left(r, \frac{1}{f^{(k)}}\right) \leq N_{p+k}\left(r, \frac{1}{f}\right)+k \bar{N}(r, f)+S(r, f) .
$$

Lemma 27. Let $f$ be a nonconstant meromorphic function, let $L$ be an $L$-function, and let $n, m$, and $k$ be three positive integers and $\alpha, \beta$ be two constants satisfying $|\alpha|+|\beta| \neq 0$. Set

$$
\begin{aligned}
& F=\left[f^{n}\left(\alpha f^{m}+\beta\right)\right]^{(k)}, \\
& G=\left[L^{n}\left(\alpha L^{m}+\beta\right)\right]^{(k)} .
\end{aligned}
$$


If $F$ and $G$ share $1 I M, n>7 k+4 \widetilde{m}+11$, then $1 /(F-1)=$ $(V G+U-V) /(G-1)$, where $U \neq 0, V$ are two constants.

Proof. Let

$$
\begin{aligned}
& W=\frac{F^{\prime \prime}}{F^{\prime}}-2 \frac{F^{\prime}}{F-1}-\left(\frac{G^{\prime \prime}}{G^{\prime}}-2 \frac{G^{\prime}}{G-1}\right), \\
& F_{1}=f^{n}\left(\alpha f^{m}+\beta\right), \\
& G_{1}=L^{n}\left(\alpha L^{m}+\beta\right) .
\end{aligned}
$$

In the same manner as Lemma 21 , we know $L$ is a nonconstant L-function. By Lemma 18, we have

$$
\begin{aligned}
& T\left(r, F_{1}\right)=(n+\widetilde{m}) T(r, f)+S(r, f), \\
& T\left(r, G_{1}\right)=(n+\widetilde{m}) T(r, L)+S(r, L) .
\end{aligned}
$$

Since $\left(F_{1}\right)^{(k)}=F$, we get

$$
m\left(r, \frac{1}{F_{1}}\right) \leq m\left(r, \frac{1}{F}\right)+S(r, f) .
$$

Suppose $W \not \equiv 0 ; z_{0}$ is a zero of $F-1$ of order $q$, and $z_{0}$ is a zero of $G-1$ of order $j$, which is possibly different from $q$, in view of $F$ and $G$ sharing 1 IM. By checking the Laurent expansion of $W$, we have $W\left(z_{0}\right)=0$ when $q=j=1$. Thus

$$
\begin{aligned}
N_{1)}\left(r, \frac{1}{F-1}\right) & =N_{1)}\left(r, \frac{1}{G-1}\right) \leq N\left(r, \frac{1}{W}\right) \\
& \leq T(r, W)+O(1) \\
& \leq N(r, W)+S(r, f)+S(r, L) .
\end{aligned}
$$

Similarly, if $z_{1}$ is a pole of $F$ of order 1 , we get $W\left(z_{1}\right) \neq \infty$. If $z_{2}$ is a pole of $G$ of order 1 , we get $W\left(z_{2}\right) \neq \infty$. Therefore, we get, by a calculation and (39), that

$$
\begin{aligned}
N(r, W) \leq & \bar{N}_{(2}(r, F)+\bar{N}_{(2}(r, G)+\bar{N}_{(2}\left(r, \frac{1}{F}\right) \\
& +\bar{N}_{(2}\left(r, \frac{1}{G}\right)+\bar{N}_{L}\left(r, \frac{1}{F-1}\right) \\
& +\bar{N}_{L}\left(r, \frac{1}{G-1}\right)+\bar{N}_{0}\left(r, \frac{1}{F^{\prime}}\right) \\
& +\bar{N}_{0}\left(r, \frac{1}{G^{\prime}}\right),
\end{aligned}
$$

where $\bar{N}_{0}\left(r, 1 / F^{\prime}\right)$ is the reduced counting function of those zeros of $F^{\prime}$ not that of $F(F-1)$. Note that $F$ and $G$ share 1 IM. We have

$$
\begin{aligned}
\bar{N}\left(r, \frac{1}{F-1}\right) \leq & N_{1)}\left(r, \frac{1}{F-1}\right)+\bar{N}_{E}^{(2}\left(r, \frac{1}{F-1}\right) \\
& +\bar{N}_{L}\left(r, \frac{1}{F-1}\right)+\bar{N}_{L}\left(r, \frac{1}{G-1}\right) .
\end{aligned}
$$

Combining (44)-(46), we get

$$
\begin{aligned}
\bar{N}(r, & \left.\frac{1}{F-1}\right)+\bar{N}\left(r, \frac{1}{G-1}\right) \\
\leq & \bar{N}_{(2}(r, F)+\bar{N}_{(2}(r, G)+\bar{N}_{(2}\left(r, \frac{1}{F}\right) \\
& +\bar{N}_{(2}\left(r, \frac{1}{G}\right)+3 \bar{N}_{L}\left(r, \frac{1}{F-1}\right) \\
& +3 \bar{N}_{L}\left(r, \frac{1}{G-1}\right)+\bar{N}_{0}\left(r, \frac{1}{F^{\prime}}\right)+\bar{N}_{0}\left(r, \frac{1}{G^{\prime}}\right) \\
& +N_{1)}\left(r, \frac{1}{G-1}\right)+2 \bar{N}_{E}^{(2}\left(r, \frac{1}{G-1}\right) .
\end{aligned}
$$

Note that

$$
\begin{aligned}
& N_{1)}\left(r, \frac{1}{G-1}\right)+2 \bar{N}_{E}^{(2}\left(r, \frac{1}{G-1}\right)+\bar{N}_{L}\left(r, \frac{1}{F-1}\right) \\
& \quad+2 \bar{N}_{L}\left(r, \frac{1}{G-1}\right) \leq N\left(r, \frac{1}{G-1}\right) \leq T(r, G) \\
& \quad+O(1) .
\end{aligned}
$$

Substituting (48) into (47), we obtain

$$
\begin{aligned}
\bar{N}(r, & \left.\frac{1}{F-1}\right)+\bar{N}\left(r, \frac{1}{G-1}\right) \\
\leq & \bar{N}_{(2}(r, F)+\bar{N}_{(2}(r, G)+\bar{N}_{(2}\left(r, \frac{1}{F}\right) \\
& +\bar{N}_{(2}\left(r, \frac{1}{G}\right)+2 \bar{N}_{L}\left(r, \frac{1}{F-1}\right) \\
& +\bar{N}_{L}\left(r, \frac{1}{G-1}\right)+\bar{N}_{0}\left(r, \frac{1}{F^{\prime}}\right)+\bar{N}_{0}\left(r, \frac{1}{G^{\prime}}\right) \\
& +T(r, G)+O(1) .
\end{aligned}
$$

By the second fundamental theorem and (49), we get

$$
\begin{aligned}
T(r, F)+T(r, G) \leq & \bar{N}(r, F)+\bar{N}(r, G)+\bar{N}\left(r, \frac{1}{F}\right) \\
& +\bar{N}\left(r, \frac{1}{G}\right)+\bar{N}_{(2}(r, F) \\
& +\bar{N}_{(2}(r, G)+\bar{N}_{(2}\left(r, \frac{1}{F}\right) \\
& +\bar{N}_{(2}\left(r, \frac{1}{G}\right)+2 \bar{N}_{L}\left(r, \frac{1}{F-1}\right) \\
& +\bar{N}_{L}\left(r, \frac{1}{G-1}\right)+T(r, G) \\
& +S(r, f)+S(r, L) .
\end{aligned}
$$


Namely,

$$
\begin{aligned}
T(r, F) \leq & N_{2}(r, F)+N_{2}(r, G)+N_{2}\left(r, \frac{1}{F}\right) \\
& +N_{2}\left(r, \frac{1}{G}\right)+2 \bar{N}_{L}\left(r, \frac{1}{F-1}\right) \\
& +\bar{N}_{L}\left(r, \frac{1}{G-1}\right)+S(r, f)+S(r, L) .
\end{aligned}
$$

Note that

$$
\begin{aligned}
N_{2}\left(r, \frac{1}{F}\right)= & N\left(r, \frac{1}{F}\right) \\
& -\left[N_{(2}\left(r, \frac{1}{F}\right)-2 \bar{N}_{(2}\left(r, \frac{1}{F}\right)\right], \\
N_{2}\left(r, \frac{1}{G}\right)= & N\left(r, \frac{1}{G}\right) \\
& -\left[N_{(2}\left(r, \frac{1}{G}\right)-2 \bar{N}_{(2}\left(r, \frac{1}{G}\right)\right] .
\end{aligned}
$$

Let $z_{3}$ be a zero of $f$ of order $i, i \geq 1$. Since $n>3 k+\widetilde{m}+6>$ $k+2, n i-k>(k+2) i-k=(i-1) k+2 i \geq 2$, we can deduce the zeros of $F$ of order $\geq 3$. Therefore,

$$
N_{(2}\left(r, \frac{1}{F}\right)-2 \bar{N}_{(2}\left(r, \frac{1}{F}\right) \geq(n-k-2) N\left(r, \frac{1}{f}\right) .
$$

Also, for $G$, we have

$$
N_{(2}\left(r, \frac{1}{G}\right)-2 \bar{N}_{(2}\left(r, \frac{1}{G}\right) \geq(n-k-2) N\left(r, \frac{1}{L}\right) .
$$

Combining (51)-(54) and the first fundamental theorem shows that

$$
\begin{aligned}
T\left(r, \frac{1}{F}\right) \leq & N_{2}(r, F)+N_{2}(r, G)+N\left(r, \frac{1}{F}\right) \\
& +N\left(r, \frac{1}{G}\right)+2 \bar{N}_{L}\left(r, \frac{1}{F-1}\right) \\
& +\bar{N}_{L}\left(r, \frac{1}{G-1}\right)-(n-k-2) N\left(r, \frac{1}{f}\right) \\
& -(n-k-2) N\left(r, \frac{1}{L}\right)+S(r, f) \\
& +S(r, L) .
\end{aligned}
$$

From (43), we see that

$$
\begin{aligned}
T\left(r, \frac{1}{F_{1}}\right) & =m\left(r, \frac{1}{F_{1}}\right)+N\left(r, \frac{1}{F_{1}}\right) \\
& \leq m\left(r, \frac{1}{F}\right)+N\left(r, \frac{1}{F_{1}}\right) \\
& =T\left(r, \frac{1}{F}\right)-N\left(r, \frac{1}{F}\right)+N\left(r, \frac{1}{F_{1}}\right) .
\end{aligned}
$$

By (55), (56), and the first fundamental theorem, we have

$$
\begin{aligned}
T\left(r, F_{1}\right) \leq & N\left(r, \frac{1}{F_{1}}\right)+N_{2}(r, F)+N_{2}(r, G) \\
& +N\left(r, \frac{1}{G}\right)+2 \bar{N}_{L}\left(r, \frac{1}{F-1}\right) \\
& +\bar{N}_{L}\left(r, \frac{1}{G-1}\right)-(n-k-2) N\left(r, \frac{1}{f}\right) \\
& -(n-k-2) N\left(r, \frac{1}{L}\right)+S(r, f) \\
& +S(r, L) .
\end{aligned}
$$

By Lemma 20, we have

$$
\begin{aligned}
N\left(r, \frac{1}{G}\right) \leq & N\left(r, \frac{1}{G_{1}}\right)+k \bar{N}\left(r, G_{1}\right)+S(r, L) \\
\leq & n N\left(r, \frac{1}{L}\right)+N\left(r, \frac{1}{\alpha L^{m}+\beta}\right) \\
& +k \bar{N}\left(r, G_{1}\right)+S(r, L) .
\end{aligned}
$$

By Lemma 26, we have

$$
\begin{aligned}
\bar{N}\left(r, \frac{1}{G}\right) \leq & N_{k+1}\left(r, \frac{1}{G_{1}}\right)+k \bar{N}\left(r, G_{1}\right)+S(r, L) \\
\leq & N_{k+1}\left(r, \frac{1}{L^{n}}\right)+N_{k+1}\left(r, \frac{1}{\alpha L^{m}+\beta}\right) \\
& +k \bar{N}\left(r, G_{1}\right)+S(r, L) \\
\leq & (k+1) \bar{N}\left(r, \frac{1}{L}\right)+N_{k+1}\left(r, \frac{1}{\alpha L^{m}+\beta}\right) \\
& +k \bar{N}\left(r, G_{1}\right)+S(r, L) .
\end{aligned}
$$

In addition, we know that

$$
\begin{aligned}
\bar{N}_{L}\left(r, \frac{1}{G-1}\right) & \leq N\left(r, \frac{1}{G-1}\right)-\bar{N}\left(r, \frac{1}{G-1}\right) \\
& \leq N\left(r, \frac{G}{G^{\prime}}\right) \leq T\left(r, \frac{G}{G^{\prime}}\right) \\
& =T\left(r, \frac{G^{\prime}}{G}\right)+O(1) \\
& \leq N\left(r, \frac{G^{\prime}}{G}\right)+m\left(r, \frac{G^{\prime}}{G}\right)+O(1) \\
& \leq \bar{N}(r, G)+\bar{N}\left(r, \frac{1}{G}\right)+S(r, L) .
\end{aligned}
$$


The same inequality (58)-(60) holds for F. Substituting (58)(60) into (57), and with (41) we deduce that

$$
\begin{aligned}
(n+\widetilde{m}) T(r, f) \leq & N_{2}(r, F)+N_{2}(r, G)+2 k \bar{N}\left(r, G_{1}\right) \\
& +2 k \bar{N}\left(r, F_{1}\right)+2 \bar{N}(r, F) \\
& +\bar{N}(r, G)+N\left(r, \frac{1}{\alpha f^{m}+\beta}\right) \\
& +N\left(r, \frac{1}{\alpha L^{m}+\beta}\right) \\
& +2 N_{k+1}\left(r, \frac{1}{\alpha f^{m}+\beta}\right) \\
& +N_{k+1}\left(r, \frac{1}{\alpha L^{m}+\beta}\right) \\
& +(3 k+4) N\left(r, \frac{1}{f}\right) \\
& +(2 k+3) N\left(r, \frac{1}{L}\right)+S(r, f) \\
& +S(r, L) .
\end{aligned}
$$

Likewise, we have

$$
\begin{aligned}
(n+\widetilde{m}) T(r, L) \leq & N_{2}(r, F)+N_{2}(r, G)+2 k \bar{N}\left(r, F_{1}\right) \\
& +2 k \bar{N}\left(r, G_{1}\right)+2 \bar{N}(r, G) \\
& +\bar{N}(r, F)+N\left(r, \frac{1}{\alpha f^{m}+\beta}\right) \\
& +N\left(r, \frac{1}{\alpha L^{m}+\beta}\right) \\
& +2 N_{k+1}\left(r, \frac{1}{\alpha L^{m}+\beta}\right) \\
& +N_{k+1}\left(r, \frac{1}{\alpha f^{m}+\beta}\right) \\
& +(3 k+4) N\left(r, \frac{1}{L}\right) \\
& +(2 k+3) N\left(r, \frac{1}{f}\right)+S(r, f) \\
& +S(r, L) .
\end{aligned}
$$

Since $L$ at most has one pole, we get $N(r, L)=O(\log r)$. At the same time, we have $\bar{N}(r, F)=\bar{N}\left(r, F_{1}\right)=\bar{N}(r, f), \bar{N}(r, G)=$ $\bar{N}\left(r, G_{1}\right)=\bar{N}(r, L), N_{2}(r, F) \leq 2 \bar{N}(r, F)$. By (61) and (62), we can obtain the following results, respectively:

$$
\begin{aligned}
(n+\widetilde{m}) T(r, f) \leq & (5 k+3 \widetilde{m}+8) T(r, f) \\
& +(2 k+2 \widetilde{m}+3) T(r, L) \\
& +S(r, f)+S(r, L),
\end{aligned}
$$

$$
\begin{aligned}
(n+\widetilde{m}) T(r, L) \leq & (4 k+2 \widetilde{m}+6) T(r, f) \\
& +(3 k+3 \widetilde{m}+4) T(r, L) \\
& +S(r, f)+S(r, L) .
\end{aligned}
$$

Assume that there exists some subset $E \subseteq R^{+}$with its linear measure mesE $=\infty$ satisfying $T(r, L) \leq T(r, f)$, as $r \in E$ and $r \longrightarrow \infty$. Then it follows from (63) that $n \leq 7 k+4 \widetilde{m}+$ 11 , which contradicts $n>7 k+4 \widetilde{m}+11$. Assume that there exists some subset $E \subseteq R^{+}$with its linear measure mes $E=\infty$ satisfying $T(r, L) \leq T(r, f)$, as $r \in E$ and $r \longrightarrow \infty$. Then it follows from (64) that $n \leq 7 k+4 \widetilde{m}+10$, which contradicts $n>7 k+4 \widetilde{m}+11$. Therefore, $W \equiv 0$. That is,

$$
\frac{F^{\prime \prime}}{F^{\prime}}-2 \frac{F^{\prime}}{F-1}=\frac{G^{\prime \prime}}{G^{\prime}}-2 \frac{G^{\prime}}{G-1} .
$$

Integrating this gives $1 /(F-1)=(V G+U-V) /(G-1)$, where $U \neq 0, V$ are two constants.

This completes the proof of Lemma 27.

Remark 28. In Theorem 11, let $F=\left[f^{n}\left(\alpha f^{m}+\beta\right)\right]^{(k)}, G=$ $\left[L^{n}\left(\alpha L^{m}+\beta\right)\right]^{(k)}$. By Lemma 27, we can get $1 /(F-1)=$ $(V G+U-V) /(G-1)$, where $U \neq 0, V$ are two constants. We can get that $F$ and $G$ share $1 \mathrm{CM}$. Then we can get Theorem 11 by Theorem 6 . Similarly, we get Theorem 12 by Theorem 9 .

\section{Proof of Theorem 6}

Let

$$
\begin{aligned}
& F=\left[f^{n}\left(\alpha f^{m}+\beta\right)\right]^{(k)}, \\
& G=\left[L^{n}\left(\alpha L^{m}+\beta\right)\right]^{(k)} .
\end{aligned}
$$

By Lemma 21, we have

$$
\frac{1}{F-1}=\frac{V G+U-V}{G-1}
$$

where $U \neq 0, V$ are two constants.

We discuss three cases.

Case $1 . V \neq 0$ and $U=V$. Then (67) can be written as

$$
\frac{1}{F-1}=\frac{V G}{G-1}
$$

Subcase 1.1. $V=-1$. From $(68)$ we have $F G=1$,

$$
\left[f^{n}\left(\alpha f^{m}+\beta\right)\right]^{(k)}\left[L^{n}\left(\alpha L^{m}+\beta\right)\right]^{(k)} \equiv 1 \text {. }
$$

Let $d_{1}$ be the degree of $L$. Then $d_{1}=2 \sum_{j=1}^{K} \lambda_{j}>0$, where $K$ and $\lambda_{j}$ are the numbers of the axiom (iii) of the definition of L-function. Thus, by Steuding [2], p.150, we get

$$
T(r, L)=\frac{d_{1}}{\pi} r \log r+O(r)
$$


Next we distinguish two cases.

Subcase 1.1.1. $\alpha \beta \neq 0$. By (69), (70), Lemma 18, and a result from Whittaker [16], p.82, we have

$$
\begin{aligned}
\rho(f) & =\rho\left(f^{n}\left(\alpha f^{m}+\beta\right)\right)=\rho\left(\left[f^{n}\left(\alpha f^{m}+\beta\right)\right]^{(k)}\right) \\
& =\rho\left(\left[L^{n}\left(\alpha L^{m}+\beta\right)\right]^{(k)}\right)=\rho\left(L^{n}\left(\alpha L^{m}+\beta\right)\right) \\
& =\rho(L)=1 .
\end{aligned}
$$

Since $L$ at most has one pole $z=1$, we deduce by (69) that $\left[f^{n}\left(\alpha f^{m}+\beta\right)\right]^{(k)}$ at most has one zero $z=1$. By (71), the assumption $k \geq 2$, and Lemma 23, we have that $f^{n}\left(\alpha f^{m}+\beta\right)$ and so $f$ has at most finitely many poles. This together with (69) implies that $\left[L^{n}\left(\alpha L^{m}+\beta\right)\right]^{(k)}$ has at most finitely many zeros. Moreover, by the assumption $n>3 k+\widetilde{m}+6$, we deduce that $L$ has at most finitely many zeros. Thus,

$$
L=Q_{1} e^{A_{1} z+B_{1}},
$$

where $Q_{1}$ is a rational function such that $L$ has neither a pole nor a zero, $A_{1} \neq 0$, and $B_{1}$ are constants. In view of (72) and Hayman [4], p.7, we have

$$
\begin{aligned}
T(r, L) & =T\left(r, Q_{1} e^{A_{1} z+B_{1}}\right) \\
& \leq \frac{\left|A_{1}\right| r}{\pi}(1+o(1))+O(\log r),
\end{aligned}
$$

which contradicts (70).

Subcase 1.1.2. $\alpha \beta=0$. By $|\alpha|+|\beta| \neq 0$, we can consider two subcases.

Subcase 1.1.2.1. $\alpha=0, \beta \neq 0$. Then (69) becomes $\left(\beta f^{n}\right)^{(k)}\left(\beta L^{n}\right)^{(k)} \equiv 1$. Assume that $z_{0}$ is a zero of $L$ of order $\lambda$. Then, we can get that $z_{0}$ is a pole of $f$ of order $\mu$, satisfying $n \lambda-k=n \mu+k$, that is, $(\lambda-\mu) n=2 k$, and we have $n \leq 2 k$, which contradicts the assumption $n>3 k+\widetilde{m}+6$. Hence, we prove that $L$ has no zeros,

$$
L=Q_{2} e^{A_{2} z+B_{2}},
$$

where $Q_{2}$ is a rational function satisfying that $L$ has no poles and $A_{2} \neq 0, B_{2}$ are two constants. In view of (74) and Hayman [4], p.7, we have

$$
\begin{aligned}
T(r, L) & =T\left(r, Q_{2} e^{A_{2} z+B_{2}}\right) \\
& \leq \frac{\left|A_{2}\right| r}{\pi}(1+o(1))+O(\log r),
\end{aligned}
$$

which contradicts (70).

Subcase 1.1.2.2. $\alpha \neq 0, \beta=0$. By using the argument as in Subcase 1.1.2.1, we obtain that $(n+\widetilde{m})(\lambda-\mu)=2 k$, and so $n \leq 2 k-\widetilde{m}$, which contradicts the assumption $n>3 k+\widetilde{m}+6$.
Hence, we know that $L$ has no zeros. Similarly, we get a contradiction.

Subcase 1.2.V $\neq-1$. Then it follows from (68) that

$$
F-\left(1+\frac{1}{V}\right)=-\frac{1}{V G}
$$

Noting that $L$ has at most one pole, then $G$ has at most one pole; from (76) we have that $F-(1+1 / V)$ has at most one zero. By Lemma 19, we obtain

$$
\begin{aligned}
(n+\widetilde{m}) T(r, f)= & T\left(r, f^{n}\left(\alpha f^{m}+\beta\right)\right)+S(r, f) \\
\leq & \bar{N}(r, f) \\
& +N_{k+1}\left(r, \frac{1}{f^{n}\left(\alpha f^{m}+\beta\right)}\right) \\
& +\bar{N}\left(r, \frac{1}{F-(1+1 / V)}\right) \\
& +S(r, f) \\
\leq & \bar{N}(r, f)+(k+1) \bar{N}\left(r, \frac{1}{f}\right) \\
& +N_{k+1}\left(r, \frac{1}{\alpha f^{m}+\beta}\right)+O(\log r) \\
& +S(r, f) \\
\leq & (k+\widetilde{m}+2) T(r, f)+S(r, f),
\end{aligned}
$$

which contradicts $n>3 k+\widetilde{m}+6$.

Case 2. $V \neq 0$ and $U \neq V$. Then from (67), we have

$$
G+\frac{U-V}{V}=\frac{-U}{V^{2}} \cdot \frac{1}{F-(1+1 / V)}
$$

Similarly, noting that $L$ has at most one pole, from (78) we have that $F-(1+1 / V)$ has at most one zero. By using the same method as in Subcase 1.2, we know it is a paradox.

Case 3. $V=0$ and $U \neq 0$. From (67) we get

$$
G=U F-U+1,
$$

so that

$$
L^{n}\left(\alpha L^{m}+\beta\right)=U f^{n}\left(\alpha f^{m}+\beta\right)+(1-U) \gamma(z),
$$

where $\gamma(z)$ is a polynomial of degree at most $k$. By (70), we get that $L$ is a transcendental meromorphic function, and so $L^{n}\left(\alpha L^{m}+\beta\right)$ is a transcendental meromorphic function. Then, we obtain $T(r, \gamma)=o\{T(r, L)\}$. If $U \neq 1$, then $(1-U) \gamma(z) \not \equiv 0$. 
Considering $L$ has at most one pole, we get from (80) and Lemma 24 that

$$
\begin{aligned}
(n+\widetilde{m}) T(r, L)= & T\left(r, L^{n}\left(\alpha L^{m}+\beta\right)\right)+S(r, L) \\
\leq & \bar{N}(r, L)+\bar{N}\left(r, \frac{1}{L^{n}\left(\alpha L^{m}+\beta\right)}\right) \\
& +\bar{N}\left(r, \frac{1}{f^{n}\left(\alpha f^{m}+\beta\right)}\right)+S(r, L) \\
\leq & \bar{N}\left(r, \frac{1}{L}\right)+\bar{N}\left(r, \frac{1}{f}\right) \\
& +\bar{N}\left(r, \frac{1}{\alpha L^{m}+\beta}\right) \\
& +\bar{N}\left(r, \frac{1}{\alpha f^{m}+\beta}\right)+O(\log r) \\
& +S(r, L) \\
\leq & (\widetilde{m}+1)[T(r, f)+T(r, L)] \\
& +S(r, L) .
\end{aligned}
$$

In addition, from (80) and Lemma 18 we have $T(r, L)=$ $T(r, f)+S(r, L)$. Using this in (81) we have

$$
(n+\widetilde{m}) T(r, L) \leq 2(\widetilde{m}+1) T(r, L)+S(r, L),
$$

we get that $n \leq \widetilde{m}+2$, a contradiction. Hence, we have $U=1$. By (80), we obtain that

$$
f^{n}\left(\alpha f^{m}+\beta\right)=L^{n}\left(\alpha L^{m}+\beta\right) .
$$

Consider two subcases as follows.

Subcase 3.1. $\alpha \beta=0$, by $|\alpha|+|\beta| \neq 0$; we deduce $f=t L$, where $t$ is a constant satisfying $t^{n+\widetilde{m}}=1$.

Subcase 3.2. $\alpha \beta \neq 0$; set $H=f / L$; by (83) we deduce

$$
-\frac{\alpha}{\beta} L^{m}\left(H^{n+m}-1\right)=H^{n}-1 .
$$

We discuss two subcases.

Subcase 3.2.1. $H$ is a constant. If $H^{n+m} \neq 1$, by (84), we get that $L$ is a constant, which contradicts the assumption that $L$ is a nonconstant L-function. Therefore, $H^{n+m}=1$, and so it follows by (84) that $H^{m}=H^{n}=1$; that is, $f^{n}=L^{n}$ and $f^{m}=L^{m}$. We get $f^{d}=L^{d}$.

Subcase 3.2.2.H is a nonconstant meromorphic function. From (84), we have

$$
-\frac{\alpha}{\beta} L^{m}=\frac{H^{n}-1}{H^{n+m}-1} .
$$

Since $L$ has at most one pole, we divide this case into two subcases again.

Subcase 3.2.2.1. $L$ has no poles. Then, from (85) we get every 1-point of $H^{n+m}$ has to be 1-point of $H^{n}$. Since $H^{n+m}=H^{n} H^{m}$, we have any 1-point of $H^{n+m}$ be a 1-point of $H^{m}$. In view of $n>$ $3 k+\widetilde{m}+6$, we deduce that $H$ is a constant, which contradicts the assumption.

Subcase 3.2.2.2.L has one and only one pole. Then, from (85) we get every zero of $H^{n+m}-1$ has to be zero of $H^{n}-1$ with one exception. Set

$$
\begin{aligned}
H^{n}-1 & =(H-1)\left(H-\delta_{1}\right) \cdots\left(H-\delta_{n-1}\right), \\
H^{n+m}-1 & =(H-1)\left(H-\eta_{1}\right) \cdots\left(H-\eta_{n+m-1}\right),
\end{aligned}
$$

where $\delta_{1}, \delta_{2}, \ldots, \delta_{n-1}$ are $n-1$ distinct finite complex numbers satisfying $\delta_{i}^{n}=1, \delta_{i} \neq 1,1 \leq i \leq n-1 ; \eta_{1}, \eta_{2}, \ldots, \eta_{n+m-1}$ are $n+m-1$ distinct finite complex numbers satisfying $\eta_{j}^{n+m}=1$, $\eta_{j} \neq 1,1 \leq j \leq n+m-1$.

Let $m=1$. From Lemma 25 we know $H^{n}-1$ and $H^{n+1}-1$ have only one common zero; then $H$ cannot be equal to any $n+m-2$ values of $\left\{\eta_{1}, \eta_{2}, \ldots, \eta_{n+m-1}\right\}$. By $n>3 k+\widetilde{m}+6$, we deduce $H$ is a constant, which contradicts the assumption.

Let $m \geq 2$. If any 1-point of $H^{n}$ is a 1-point of $H^{n+m}$, then any 1-point of $H^{n}$ is a 1-point of $H^{m}$, since $n>3 k+\widetilde{m}+6$, a contradiction to the assumption that $H$ is nonconstant. If there is at least one $\delta_{i} \neq \eta_{j}, 1 \leq i \leq n-1,1 \leq j \leq n+m-1$, then $H$ cannot be equal to any $m+1$ values of $\left\{\eta_{1}, \eta_{2}, \ldots, \eta_{n+m-1}\right\}$. By $m \geq 2$, we deduce $H$ is a constant, which contradicts the assumption.

This completes the proof of Theorem 6 .

\section{Proof of Theorem 9}

Set

$$
\begin{aligned}
F & =\left[f^{n}(f-1)^{m}\right]^{(k)}, \\
G & =\left[L^{n}(L-1)^{m}\right]^{(k)}, \\
F_{1} & =f^{n}(f-1)^{m}, \\
G_{1} & =L^{n}(L-1)^{m} .
\end{aligned}
$$

By Lemma 18, we get

$$
\begin{aligned}
& T\left(r, F_{1}\right)=(n+m) T(r, f)+S(r, f), \\
& T\left(r, G_{1}\right)=(n+m) T(r, L)+S(r, L) .
\end{aligned}
$$

By Lemma 21, we have

$$
\frac{1}{F-1}=\frac{V G+U-V}{G-1},
$$

where $U \neq 0$ and $V$ are two constants.

We discuss three cases.

Case 1. $V \neq 0$ and $U=V$. By (91), we have

$$
\frac{1}{F-1}=\frac{V G}{G-1} \text {. }
$$

Subcase 1.1. $V=-1$; then from (92) $F G=1$,

$$
\left[f^{n}(f-1)^{m}\right]^{(k)}\left[L^{n}(L-1)^{m}\right]^{(k)} \equiv 1 .
$$


Let $d_{1}$ be the degree of $L$. Then $d_{1}=2 \sum_{j=1}^{K} \lambda_{j}>0$, where $K$ and $\lambda_{j}$ are the numbers of the axiom (iii) of the definition of L-function. Thus, by Steuding [2], p150, we get

$$
T(r, L)=\frac{d_{1}}{\pi} r \log r+O(r) .
$$

By (93), (94), Lemma 18, and a result from Whittaker [16], p.82, we have

$$
\begin{aligned}
\rho(f) & =\rho\left(f^{n}(f-1)^{m}\right)=\rho\left(\left[f^{n}(f-1)^{m}\right]^{(k)}\right) \\
& =\rho\left(\left[L^{n}(L-1)^{m}\right]^{(k)}\right)=\rho\left(L^{n}(L-1)^{m}\right) \\
& =\rho(L)=1 .
\end{aligned}
$$

Since $L$ at most has one pole $z=1$, we deduce by (93) that $\left[f^{n}\left(\alpha f^{m}+\beta\right)\right]^{(k)}$ at most has one zero $z=1$. By (95), the assumption $k \geq 2$, Lemma 23 , we get $f^{n}(f-1)^{m}$ and so $f$ has at most finitely many poles. This together with (93) implies that $\left[L^{n}(L-1)^{m}\right]^{(k)}$ has at most finitely many zeros. Moreover, by the assumption $n>3 k+m+6$, we deduce that $L$ has at most finitely many zeros. Thus,

$$
L=Q e^{A z+B},
$$

where $Q$ is a rational function satisfying $L$ has no zeros and poles, $A \neq 0$, and $B$ are constants. By (96) and Hayman [4], we get

$$
\begin{aligned}
T(r, L) & =T\left(r, Q e^{A z+B}\right) \\
& \leq \frac{|A| r}{\pi}(1+o(1))+O(\log r) .
\end{aligned}
$$

which contradicts (94).

Subcase 1.2. $V \neq-1$; then from (92) we have

$$
F-\left(1+\frac{1}{V}\right)=-\frac{1}{V G} \text {. }
$$

In view of $L$ having at most one pole $z=1$, we know that $G$ has at most one pole $z=1$, from (98) we have that $F-(1+1 / V)$ has at most one zero. By (89) and Lemma 19 we obtain

$$
\begin{aligned}
(n+m) T(r, f)= & T\left(r, F_{1}\right)+S(r, f) \\
\leq & \bar{N}(r, f)+N_{k+1}\left(r, \frac{1}{F_{1}}\right) \\
& +\bar{N}\left(r, \frac{1}{F-(1+1 / V)}\right) \\
& +S(r, f) \\
\leq & \bar{N}(r, f)+(k+1) N\left(r, \frac{1}{f}\right) \\
& +N_{k+1}\left(r, \frac{1}{(f-1)^{m}}\right) \\
& +O(\log r)+S(r, f) \\
\leq & (k+m+2) T(r, f)+S(r, f),
\end{aligned}
$$

which contradicts $n>3 k+m+6$.
Case 2. $V \neq 0$ and $U \neq V$. From (91), we have

$$
G+\frac{U-V}{V}=\frac{-U}{V^{2}} \cdot \frac{1}{F-(1+1 / V)} .
$$

Similarly, noting that $L$ has at most one pole $z=1$, by (100) we have that $F-(1+1 / V)$ has at most one zero. By using the same method as in Subcase 1.2, we know it is a paradox.

Case 3. $V=0$ and $U \neq 0$. Then, from (91), we have

$$
G=U F-U+1,
$$

so that

$$
G_{1}=U F_{1}+(1-U) \gamma(z)
$$

where $\gamma(z)$ is a polynomial of degree at most $k$. By (94), we get $L$ is a transcendental meromorphic function, and so $G_{1}$ is a transcendental meromorphic function. Then, we get $T(r, \gamma)=$ $o\{T(r, L)\}$. If $\gamma \neq 1$, then $(1-U) \gamma(z) \neq \equiv 0$. From (102) and Lemma 24 we have

$$
\begin{aligned}
(n+m) T(r, L)= & T\left(r, G_{1}\right)+S(r, L) \\
\leq & \bar{N}\left(r, G_{1}\right)+\bar{N}\left(r, \frac{1}{G_{1}}\right) \\
& +\bar{N}\left(r, \frac{1}{G_{1}-(1-U) P(z)}\right) \\
& +S(r, L) \\
= & \bar{N}(r, L)+\bar{N}\left(r, \frac{1}{G_{1}}\right) \\
& +\bar{N}\left(r, \frac{1}{F_{1}}\right)+S(r, L) \\
\leq & \bar{N}\left(r, \frac{1}{L}\right)+\bar{N}\left(r, \frac{1}{f}\right) \\
& +\bar{N}\left(r, \frac{1}{L-1}\right)+\bar{N}\left(r, \frac{1}{f-1}\right) \\
& +O(\log r)+S(r, L) \\
\leq & 2[T(r, f)+T(r, L)]+S(r, L) .
\end{aligned}
$$

In addition, by (102) and Lemma 18 we have $T(r, L)=$ $T(r, f)+S(r, L)$. Using this in (103) we have

$$
(n+m) T(r, L) \leq 4 T(r, L)+S(r, L),
$$

which contradicts $n>3 k+m+6$. Hence, we get $U=1$. By (102), we obtain $F_{1}=G_{1}$; that is,

$$
f^{n}(f-1)^{m}=L^{n}(L-1)^{m} .
$$

That is,

$$
\begin{aligned}
& f^{n}\left(f^{m}+\cdots+(-1)^{i} C_{m}^{m-i} f^{m-i}+\cdots+(-1)^{m}\right) \\
& \quad=L^{n}\left(L^{m}+\cdots+(-1)^{i} C_{m}^{m-i} L^{m-i}+\cdots+(-1)^{m}\right) .
\end{aligned}
$$


Set $H=f / L$. If $H$ is a nonconstant meromorphic function, from (105) we get $f^{n}(f-1)^{m} \equiv L^{n}(L-1)^{m}$. If $H$ is a constant, from (106) we get

$$
\begin{aligned}
L^{n+m} & \left(H^{n+m}-1\right)+\cdots \\
& +(-1)^{i} C_{m}^{m-i} L^{n+m-i}\left(H^{n+m-i}-1\right)+\cdots \\
& -L^{n}\left(H^{n}-1\right)=0 .
\end{aligned}
$$

which implies $H=1$. Therefore, $f \equiv L$.

This completes the proof of Theorem 9.

\section{Data Availability}

The data used to support the findings of this study are included within the article.

\section{Conflicts of Interest}

The authors declare that there are no conflicts of interest regarding the publication of this paper.

\section{Acknowledgments}

Project was supported by the National Natural Science Foundation of China (Grant no. 11301076), the Natural Science Foundation of Fujian Province, China (Grant no. 2018J01658), and Key Laboratory of Applied Mathematics of Fujian Province University (Putian University) (Grant no. SX201801).

\section{References}

[1] J. Kaczorowski and A. Perelli, "On the structure of the Selberg class. I. $0 \leq \mathrm{d} \leq 1$," Acta Mathematica, vol. 182, no. 2, pp. 207-241, 1999.

[2] J. Steuding, Value-distribution of L-functions, Springer, Berlin, 2007.

[3] P. C. Hu and B. Q. Li, "A simple proof and strengthening of a uniqueness theorem for L-functions," Canadian Mathematical Bulletin, vol. 59, no. 1, pp. 119-122, 2016.

[4] W. K. Hayman, Meromorphic Functions, Clarendon Press, Oxford, UK, 1964.

[5] H. X. Yi and C. C. Yang, Uniqueness Theory of Meromorphic Functions, Kluwer Academic Publishers, Beijing, China, 2003.

[6] B. Q. $\mathrm{Li}$, "A result on value distribution of $L$-functions," Proceedings of the American Mathematical Society, vol. 138, no. 6, pp. 2071-2077, 2010.

[7] I. Lahiri, "Uniqueness of meromorphic functions as governed by their differential polynomials," Yokohama Mathematical Journal, vol. 44, pp. 147-156, 1997.

[8] M. L. Fang, "Uniqueness and value-sharing of entire functions," Computers \& Mathematics with Applications. An International Journal, vol. 44, no. 5-6, pp. 823-831, 2002.

[9] F. Liu, X. M. Li, and H. X. Yi, "Value distribution of L-functions concerning shared values and certain differential polynomials," Proceedings of the Japan Academy, Series A, Mathematical Sciences, vol. 93, no. 5, pp. 41-46, 2017.
[10] F. Liu, X. M. Li, and H. X. Yi, Results on L-functions whose certain differential polynomials share one finite nonzero value, to appear.

[11] A. Z. Mokhonko, "On the Nevanlinna characteristics of some meromorphic functions, in Theory of Functions, Functional Analysis and their Applications, 14," Izd-vo Kharkovsk. Un-ta, pp. 83-87, 1971.

[12] J. K. Langley, "The second derivative of a meromorphic function of finite order," Bulletin of the London Mathematical Society, vol. 35, no. 1, pp. 97-108, 2003.

[13] Q. C. Zhang, "Meromorphic functions sharing three values," Indian Journal of Pure and Applied Mathematics, vol. 30, no. 7, pp. 667-682, 1999.

[14] J. L. Zhang and L. Z. Yang, "Some results related to a conjecture of R. Brück," Journal of Inequalities in Pure and Applied Mathematics, vol. 8, no. 1, article 18, pp. 1-11, 2007.

[15] I. Lahiri and A. Sarkar, "Uniqueness of a meromorphic function and its derivative," Journal of Inequalities in Pure and Applied Mathematics, vol. 5, no. 1, Article 20, 9 pages, 2004.

[16] J. M. Whittaker, "The order of the derivative of a meromorphic function," Journal of the London Mathematical Society, vol. 11, no. 2, pp. 82-87, 1936. 


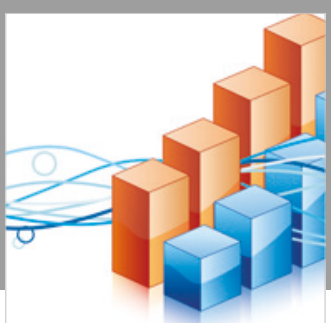

Advances in

Operations Research

\section{-n-m}
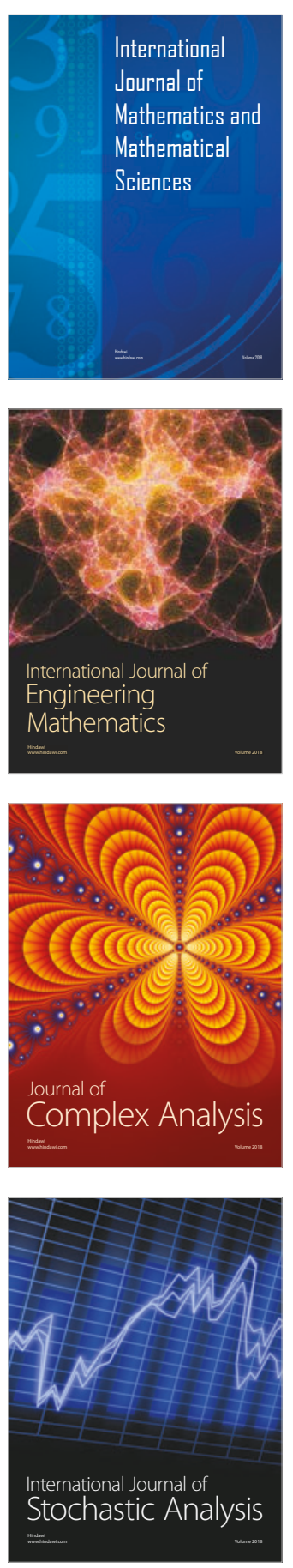
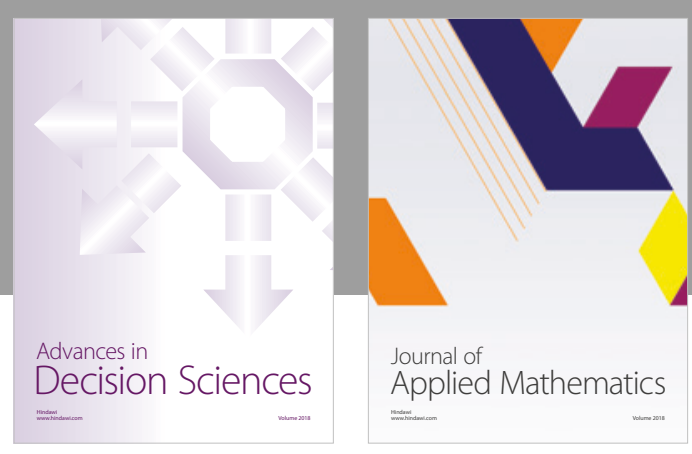

Journal of

Applied Mathematics
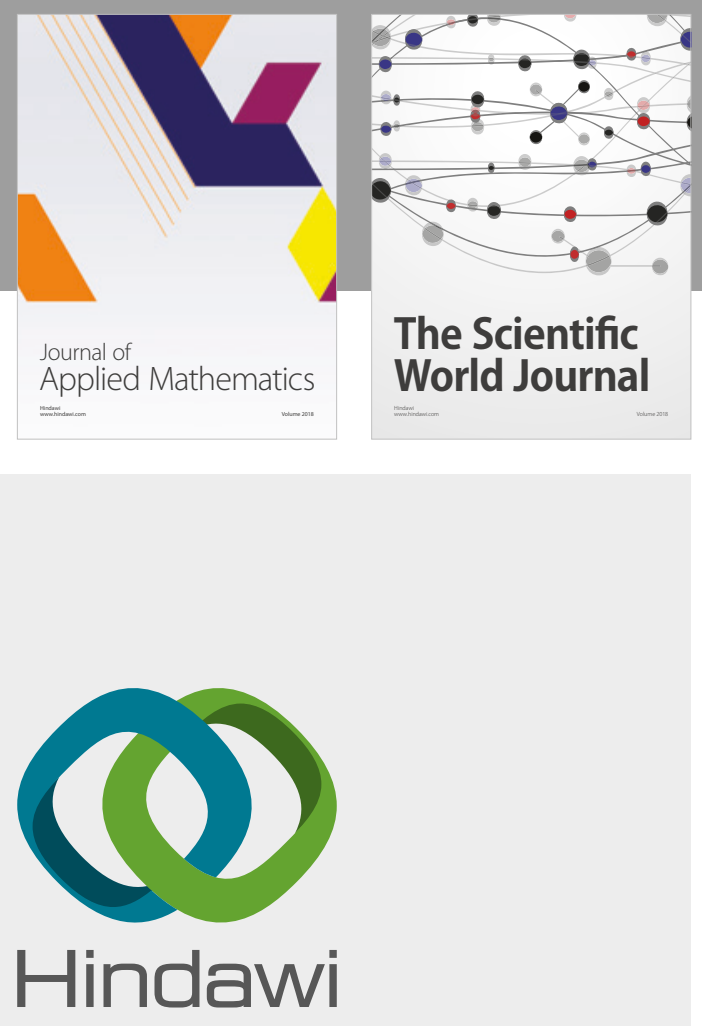

Submit your manuscripts at

www.hindawi.com

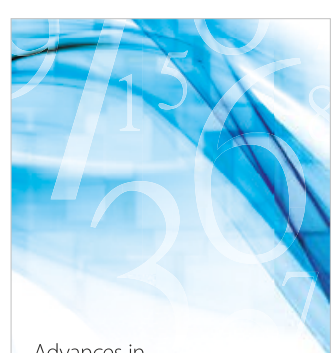

Advances in
Numerical Analysis
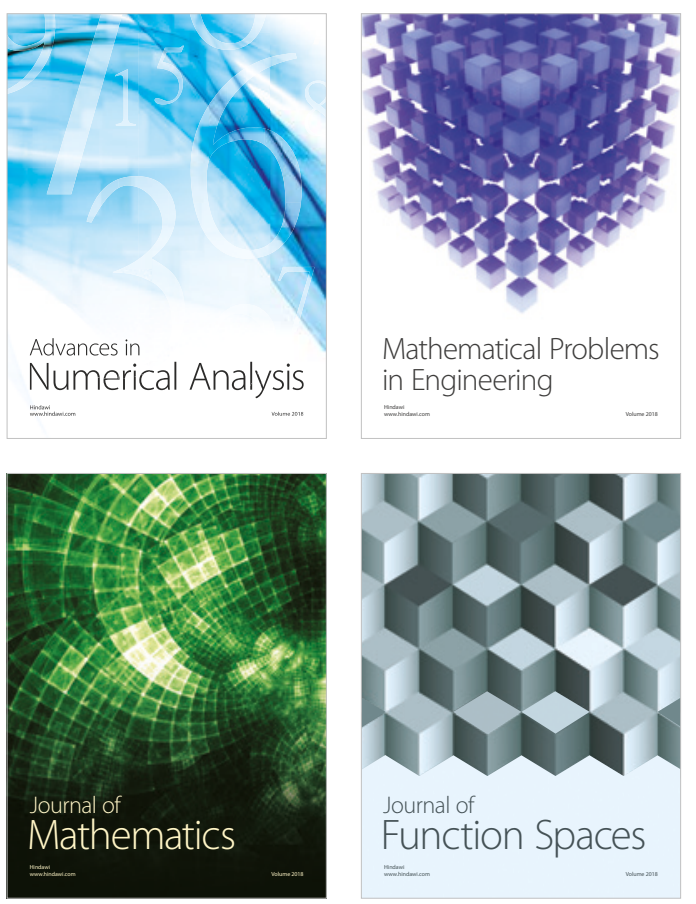

Mathematical Problems in Engineering

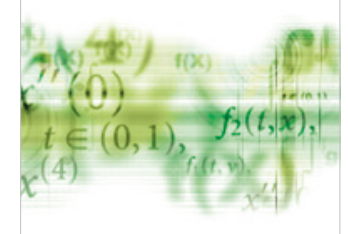

International Journal of

Differential Equations

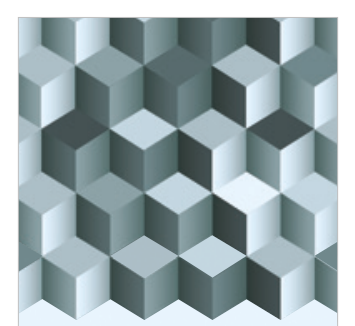

Journal of

Function Spaces
The Scientific

World Journal

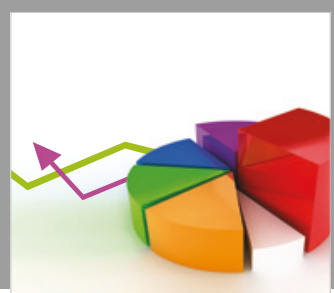

Journal of

Probability and Statistics
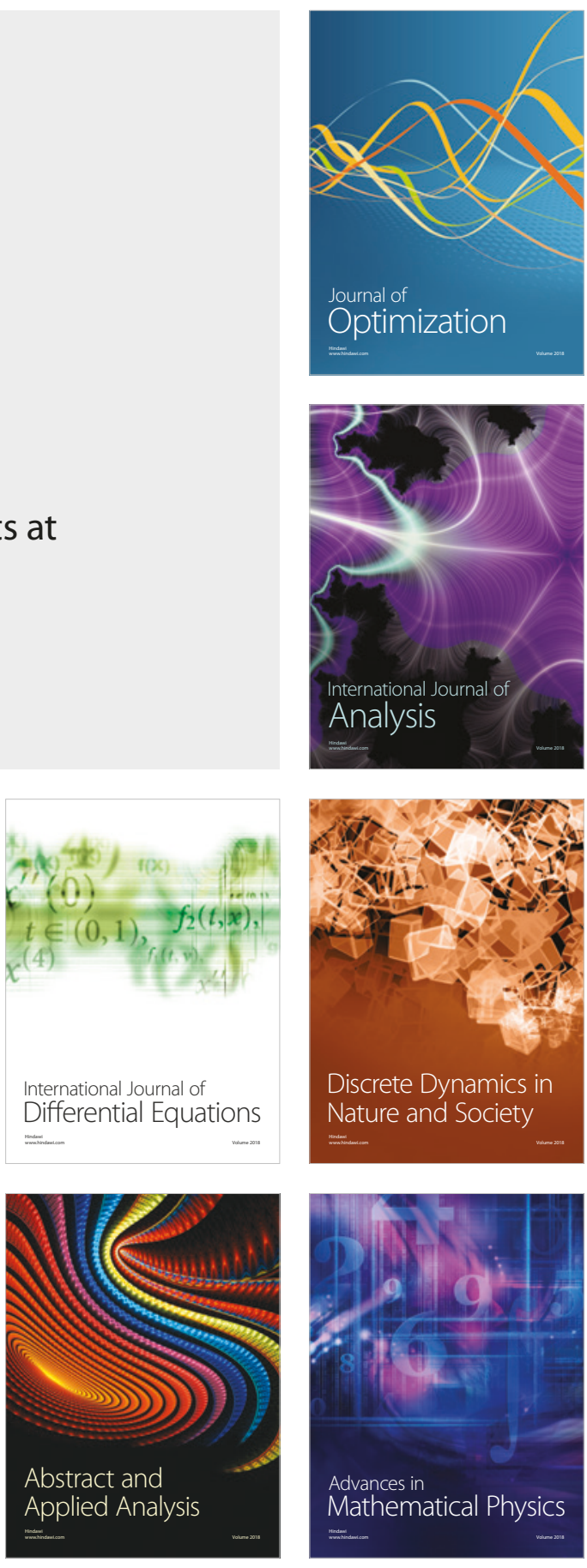